\title{
Virtual Stent Grafting in Personalized Surgical Planning for Treatment of Aortic Aneurysms Using Image-Based Computational Fluid Dynamics
}

\author{
Guanglei Xiong ${ }^{1}$ and Charles A. Taylor ${ }^{2}$ \\ ${ }^{1}$ Biomedical Informatics Program, Stanford University, CA, USA \\ ${ }^{2}$ Departments of Bioengineering and Surgery, Stanford University, CA, USA \\ \{glxiong, taylorca\} estanford.edu
}

\begin{abstract}
Image-based computational fluid dynamics provides great promise for evaluation of vascular devices and assessment of surgical procedures. However, many previous studies employ idealized arterial and device models or patientspecific models with a limited number of cases, since the model construction process is tedious and time-consuming. Moreover, in contrast to retrospective studies from existing image data, there is a pressing need of prospective analysis with the goal of surgical planning. Therefore, it is necessary to construct models with implanted devices in a fast, virtual and interactive fashion. The goal of this paper is to develop new geometric methods to deploy stent grafts virtually to patient-specific models constructed from direct 3D segmentation of medical images. A triangular surface representing vessel lumen boundary is extracted from the segmentation. The diseased portion is then clipped and replaced by the surface of a virtual stent graft following the centerline obtained from the clipped portion. A Y-shape stent graft is employed in case of bifurcated arteries. A method to map a 2D strut pattern on the stent graft is also presented. We demonstrate the application of our methods to quantify wall shear stresses and forces acting on stent grafts in personalized surgical planning for endovascular treatment of thoracic and abdominal aortic aneurysms. Our approach enables prospective model construction and may help to increase its throughput required by routine clinical uses in the future.
\end{abstract}

Keywords: model construction, geometric processing, surgical planning, stent graft, aortic aneurysm, computational fluid dynamics.

\section{Introduction}

Image-based computational fluid dynamics, once emerged, is widely used as a valuable tool for investigating the role of local hemodynamics and the development of vascular diseases, such as atherosclerosis and aneurysms [1, 2]. Nowadays, increasing attention has been focused on applications of this tool to evaluate the safety and efficacy of vascular devices [3] and assess the outcomes of surgical procedures [4]. Considerable insights were gained by studying flow through stents in occlusive [5] and aneurysmal diseases [6] and computing fluid forces on stent grafts [7]. However, 
many early studies employ idealized arterial and device models, which may be inadequate for replicating realistic hemodynamic conditions. Some recent studies have shifted to construct and analyze patient-specific models [8, 9], which provide great promises for inferring realistic conditions on an individual basis. Nevertheless, a majority of previous studies employ a limited number of cases stemming from the fact that the model construction process from 3D medical images is still tedious. Furthermore, in contrast to retrospective studies of post-operative states from existing image data, there is an obvious requirement of prospective analysis to predict the benefits or harms of a particular procedure in surgical planning.

To date, there are two major approaches to construct vascular models from medical images. One is the NURBS (Non-uniform Rational Basis Spline)-based modeling [10]. Usually, one parametric surface is reconstructed from sweeping a series of contours by segmenting in 2D over cross sectional images along the length of a vessel branch. Surfaces for multiple branches are then lofted together to represent the entire vascular tree of interest. The other is the triangle-based (sometimes polygon-based) modeling, where 3D segmentation is directly applied and triangulated into a single surface [11]. The former approach preceded the latter and remains quite useful for small vessels in images of poor contrast. However, it has been considered to be less favorable to the latter, which is more efficient, less subjective, and of more fidelity, especially for large arteries in decent-quality images. On the other hand, The NURBS-based approach yet shows great advantages in the case of surgical planning that not only arteries but also implanted devices are simultaneously modeled and ultimately combined [12]. This benefit is due to the fact that NURBS, a common representation for medical devices, offers a wealth of inherent features, such as interactive editing and Boolean operations, of which the triangle-based approach lacks. In order to fill the gap between triangle-based modeling and surgical planning, there is a pressing need for developments of new geometry processing techniques that are robust, efficient, and ideally usable in clinical settings.

In this paper, we present novel geometric methods to deploy stent grafts virtually to triangle-based patient-specific models constructed from direct 3D segmentation of medical images. Both unbranched and bifurcated stent grafts are produced for repairing thoracic and abdominal aortic aneurysms, respectively. Our approach allows the user to modify the preoperative model interactively to incorporate the effects of a virtual stent graft. We also describe a method to map a strut pattern defined on a $2 \mathrm{D}$ image onto the stent graft. Note we are here interested in the geometric effects of a deployed state rather than the deployment process itself, which takes account of mechanical interaction between stent graft and vessel wall. To demonstrate, we explore, using computational fluid dynamics, the wall shear stresses of pre-operative and virtual post-operative models as well as forces acting on stent grafts. They are informative to assess outcomes for endovascular treatment of aortic aneurysms.

\section{Methods}

The images used in this paper are from de-identified pre-existing imaging studies at Stanford University Medical Center. The image of a thoracic aorta was acquired using CT (resolution: $0.58 \times 0.58 \times 0.7 \mathrm{~mm}$, size: $512 \times 512 \times 375$ ). The image of an abdominal aorta was acquired using MRI (resolution: $0.78 \times 0.78 \times 1.5 \mathrm{~mm}$, size: $512 \times 512 \times 124$ ). 


\subsection{Geometric Model Construction and Centerline Extraction}

After the image was preprocessed, the vessel lumen was segmented using regionbased and edge-based level set methods [13]. A triangular surface mesh was extracted from the segmentation using the method proposed in [14], which produces provably good sampling and meshing. Geometric processing techniques, such as smoothing, trimming, pruning, circularization, and elongation, were performed to yield a suitable model. A method to extract the multi-branch centerline is then used. Two distance transforms of the model: distance to inlet/outlet and distance to vessel wall, were computed using the fast marching method [15]. Using the former distance, the model was divided. Centerline nodes with the maximum of the latter distance in every region were selected and connected to yield a raw centerline using adjacency relations among regions. The centerline was then refined and resampled to reach a higher quality. The detailed algorithms were reported elsewhere in [16]. Figure 1 shows the image of the abdominal aorta with an aneurysm, its geometric model and centerline.
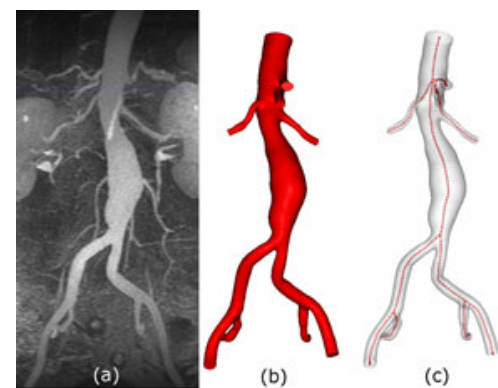

(c)

Fig. 1. An image of abdominal aorta (a), the geometric model (b), and its centerline (c).

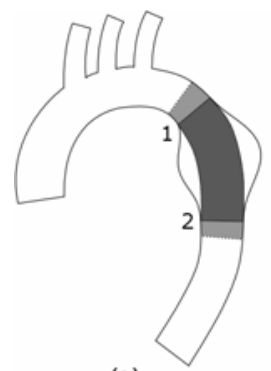

(a)

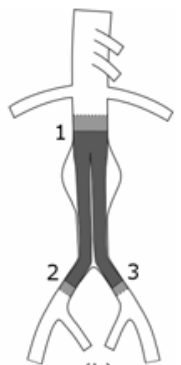

(b)

Fig. 2. Illustration of stent grafts for thoracic aortic aneurysm (a) and abdominal aortic aneurysm (b).

\subsection{Virtual Stent Grafting for Thoracic Aortic Aneurysm}

An illustration of the virtual stent graft for a model of thoracic aortic aneurysm is shown in Fig. 2(a). The aneurysm (the bulged region) is replaced by a tubular structure (in gray) connecting the proximal and distal portions of the aorta. The lighter gray regions depict fixation zones, where the graft is attached to the vessel wall. The requirements of the modified model are: (a) is a "water-tight" triangular mesh; (b) has the graft inside the original model and following a centerline; (c) keeps the portions other than the aneurysm intact; (d) has smooth transitions in fixation zones. We propose a novel algorithm to solve this problem:

(1) Trim the model at proximal and distal ends of the aneurysm (boundaries of light and dark gray regions at locations 1 and 2). It leads to two open contours, which are then circularized.

(2) Morph the surface at light gray regions by weighting the original surface with a perfectly tubular shape. The surface is weighted more to the original as further from the dark gray region. 
(3) Extract the centerline of the portion between locations 1 and 2. Our algorithm in Section 2.1 ensures the starting and ending nodes are centers of contours in step (1).

(4) Construct a tubular structure as the stent graft by creating a series of circular triangle strips between neighboring pairs of contours, guided by the centerline from step (3). We here make the radius and number of vertices linearly varied from location 1 to 2. To establish the correspondences between vertices, a starting vertex needs to be identified on either neighboring contour. In Fig. 3, $s$ and $c$ denote a starting vertex and a centerline node at the current contour, respectively. $\vec{n}_{k-1}=\left(c_{k}-c_{k-1}\right) /\left\|c_{k}-c_{k-1}\right\|$ is the normal of the previous contour. $\overrightarrow{\boldsymbol{x}}, \overrightarrow{\boldsymbol{y}}$ are local axes with $\overrightarrow{\boldsymbol{n}} \perp \overrightarrow{\boldsymbol{x}}, \overrightarrow{\boldsymbol{y}}$ and $\vec{x}=(s-c) /\|s-c\|$. The starting vertex of the next contour $s_{k}$ can be determined by $\boldsymbol{s}_{k}=\boldsymbol{c}_{k}+r_{k} \overrightarrow{\boldsymbol{n}}_{k-1} \times \overrightarrow{\boldsymbol{x}}_{k-1} \times \overrightarrow{\boldsymbol{n}}_{k}$, where $r_{k}$ is the radius. The starting vertex of the initial contour at location 1 is arbitrarily selected. The starting vertex of the final contour at location 2 is the vertex closest to that of the previous contour.

The results of our algorithm on the model of thoracic aorta are illustrated in Fig. 4.

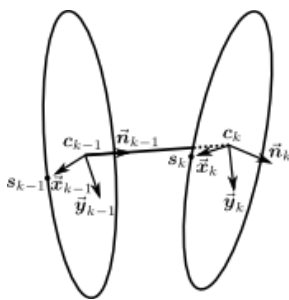

Fig. 3. Geometry of two neighboring contours to create a triangle strip.
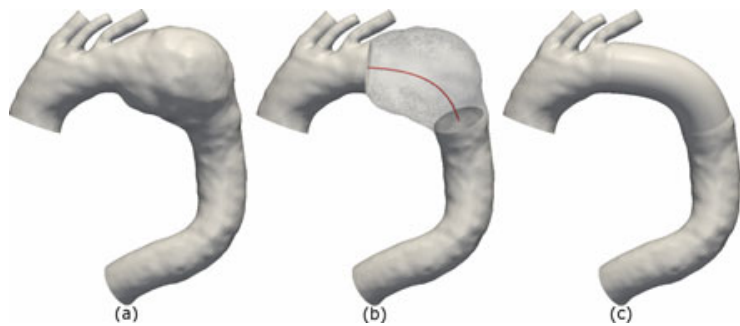

Fig. 4. Virtual stent grafting for thoracic aortic aneurysm. (a) The pre-operative model; (b) Aneurysm removal and the centerline; (c) The post-operative model.

\subsection{Virtual Stent Grafting for Abdominal Aortic Aneurysm}

In case of abdominal aortic aneurysm, the stent graft may be in $\mathrm{Y}$ shape as shown in Fig. 2(b). A special treatment of mesh shape close to the bifurcation is needed. We extend our algorithm as follows:

(1) Trim the model at the proximal end of the aneurysm (location 1) and at distal iliac arteries (locations 2 and 3). Morph the surfaces as before.

(2) Extract the centerline of the portion between locations 1, 2, and 3. Split the centerline inside the aneurysm corresponding to two iliac branches. It leads to three centerline segments

(3) Extend the surfaces along the centerline segments using tubular structures towards the bifurcation similar to before.

(4) Construct a transition surface at the bifurcation among three ending contours from last step. The transition essentially consists of a series of contours that varies from one circle in the parent vessel to two circles in the child vessels. Figure 5(a)-(d) lists four typical contours in the order from parent vessel to the child vessels. Finally, triangle strips between neighboring pairs of contours are generated to complete the transition.

The results on the model of abdominal aorta are illustrated in Fig. 6(a)-(d). Figure 6(e), (f) demonstrate the mesh at the bifurcation before and after surface transition. 


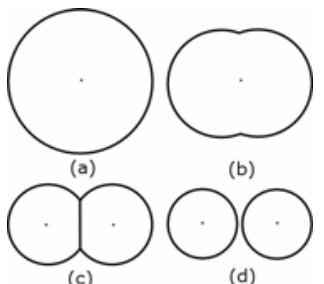

Fig. 5. Four typical contours for the surface transition at the bifurcation.

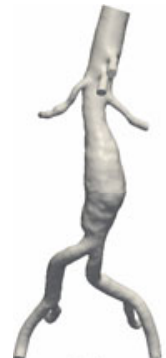

(a)

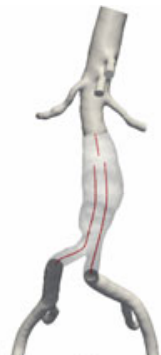

(b)

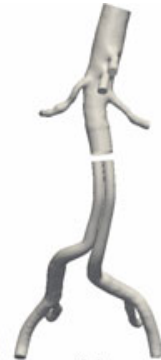

(c)

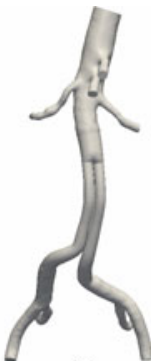

(d)

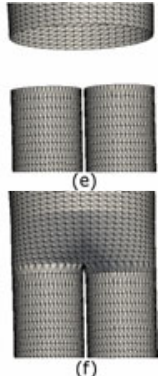

(f)

Fig. 6. Virtual stent grafting for abdominal aortic aneurysm. (a) The pre-operative model; (b) Aneurysm removal and the centerlines; (c) Surface extension; (d) The post-operative model; Mesh at the bifurcation before (e) and after (f) surface transition.

\subsection{Strut Pattern Mapping}

To make the stent graft more realistic, a particular strut pattern can be placed on the modified model to replicate the effects of a deployed stent graft. The designed stent pattern is usually described as a $2 \mathrm{D}$ image. Therefore, there is a need to map the pattern in $2 \mathrm{D}$ rectangular coordinate to $3 \mathrm{D}$ cylindrical coordinate. We assume that the struts can follow any bends in the graft, which is a first-order approximation. In addition, a typical strut width (e.g. $0.5 \mathrm{~mm}$ ) is generally smaller than the triangle size (e.g. $1.0 \mathrm{~mm}$ ) of the surface mesh, mesh refinement is necessary to represent the shape of the strut. Instead of refining the stent graft portion globally, a method to refine the mesh locally along the strut path is favorable to avoid excessively increasing the number of mesh elements and thus the computational cost. Furthermore, vertices along the strut path are displaced according to the intensity in the 2D image which characterizes specified strut thickness. Notice that strut is modeled as concavity on the surface since the stent is normally located inside the graft and the geometric model represents the channel of blood flow.

We propose a two-way algorithm that refines mesh first and then displaces vertices:

(1) Locally refine the triangles on the virtual stent graft along the strut path using a $\sqrt{3}$-subdivision scheme [17]. To ensure a full coverage of the stent graft, we define the mapping inversely as a 3D cylindrical coordinate $(r, \phi, z)$ to a $2 \mathrm{D}$ rectangular coordinate $(x, y)$ in image domain: $x=\frac{\phi}{2 \pi} W, y=\frac{z}{L} H$, where $W, H, L$ are the image width, height, and the total centerline length, respectively. A triangle is chosen to be refined if and only if its map in the image domain overlaps with the strut pattern. Since the strut pattern is sparse in the image, we first check whether there is any strut pixel in the bounding box of the triangle. If so, we further check whether the pixel region intersects the triangle.

(2) Displace vertices on the strut path radially towards the centerline by a distance depending on the intensity of its map in the $2 \mathrm{D}$ image. The linear interpolation of pixel intensity is used since maps are usually at subpixel locations.

(3) Smooth the mesh along the strut path using a diffusion-based method [18]. 


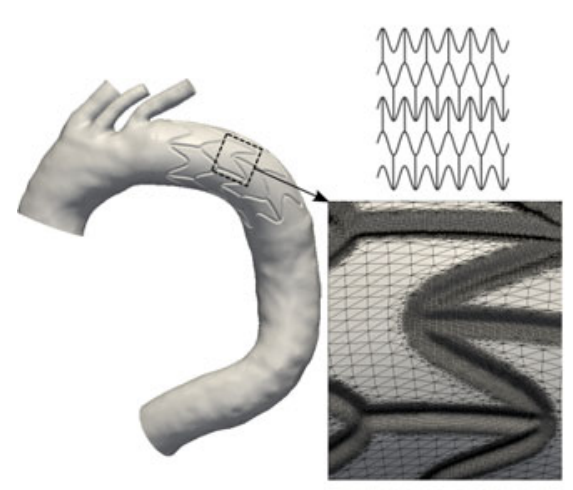

(a)

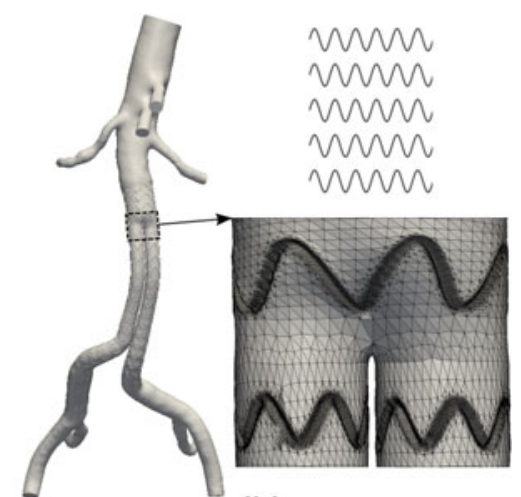

(b)

Fig. 7. Strut pattern mapping for the models of thoracic aorta (a) and abdominal aorta (b)

The results on strut pattern mapping are illustrated in Fig. 7 with given patterns and mesh details along the strut paths.

\section{Simulation Results}

Three models (pre-operative, post-operative without and with strut) were discretized into tetrahedral meshes for thoracic aorta $(0.92 \mathrm{M}, 0.66 \mathrm{M}, 2.05 \mathrm{M})$ and abdominal aorta $(1.83 \mathrm{M}, 1.57 \mathrm{M}, 2.80 \mathrm{M})$. Blood flow was solved using a custom stabilized finite element solver developed in our group [19]. Five cardiac cycles were simulated with assumptions of rigid wall and Newtonian fluid behavior. All results presented were from the last and converged cycle.

In Fig. 8, we show the mean wall shear stress (MWSS) of the three models, which is computed as the time-averaged amplitude of shear stress on the wall. For both cases, low MWSS are observed in the aneurysm regions. After virtual stent grafts are deployed, MWSS is higher in the same regions due to reduced calibers and increased blood velocity. This suggests stent grafts may help to reduce recirculation and their design should include the consideration of the raised levels of shear stress. In addition, strut patterns apparently influence local shear stress in vicinity. Therefore, it is necessary to include the modeling of strut to accurately quantify shear stress.

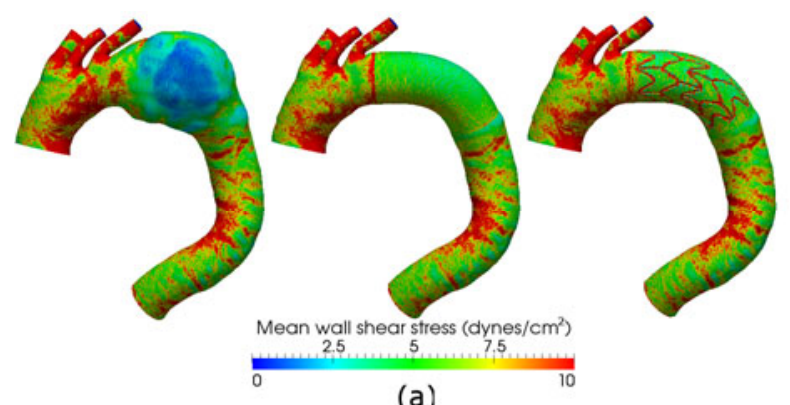

(a)

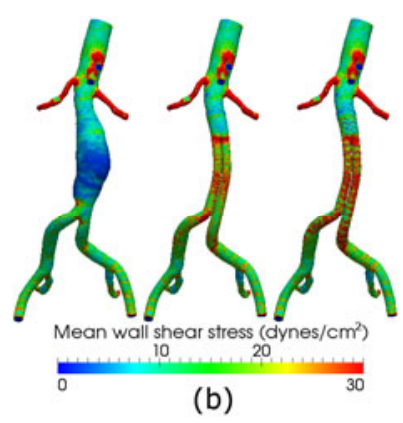

(b)

Fig. 8. Mean wall shear stress for the models of thoracic aorta (a) and abdominal aorta (b) 


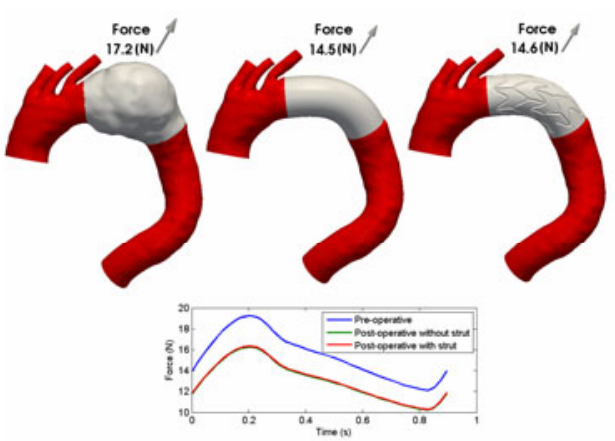

(a)

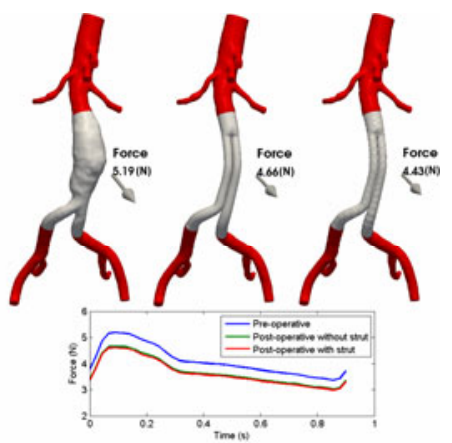

(b)

Fig. 9. Forces on aneurysm and stent graft for the models of thoracic aorta (a) and abdominal aorta (b).

The forces acting on the aneurysmal walls and stent grafts are believed to have causal effects of aneurysm rupture and stent graft migration. In Fig. 9, we show these forces on the aneurysm and stent graft (gray regions), which are calculated as the surface integral of tensile and shear forces. The arrows depict the forces at peak systole. In both cases, the forces are directed radially outwards with respect to curved paths due to the area difference between interior and exterior led by the curvature. The magnitude of the force is smaller in the stent graft than that in the aneurysm due to reduced areas. This holds true for the whole cardiac cycle as shown in the plots. The strut patterns do not have a significant impact on the magnitudes of the forces as their contributions to the surface integration are minimal.

\section{Discussion and Conclusion}

In this paper, we described new geometric methods to construct virtual post-operative models for simulation-based personalized planning of stent grafting of aortic aneurysms. The methods fill the gap between triangle-based modeling and prospective analysis. Our experiences show it normally takes less than ten minutes to construct the post-operative model from a pre-operative model. Our approach thus shows promises for its future use in clinical settings. We demonstrated simulations results of wall shear stress and force acting on stent grafts.

Our approach is generalizable to handle other models of vascular diseases, such as aortic coarctation, coronary stenosis, and cerebral aneurysms. Future works will focus on testing robustness and efficiency of our methods on a large number of cases and performing validations using experimental studies and some evaluations on dealing with more challenging cases. We aim at providing a user-friendly simulation tool for vascular surgeons to plan interventions.

\section{References}

1. Steinman, D.A., Taylor, C.A.: Flow Imaging and Computing: Large Artery Hemodynamics. Annals of Biomedical Engineering 33, 1704-1709 (2005)

2. Taylor, C.A., Steinman, D.A.: Image-Based Modeling of Blood Flow and Vessel Wall Dynamics: Applications, Methods and Future Directions. Annals of Biomedical Engineering (2010) 
3. Zarins, C.K., Taylor, C.A.: Endovascular Device Design in the Future: Transformation from Trial and Error to Computational Design. Journal of Endovascular Therapy 16(suppl. 1), I12-21 (2009)

4. Taylor, C.A., Draney, M.T., Ku, J.P., Parker, D., Steele, B.N., Wang, K., Zarins, C.K.: Predictive Medicine: Computational Techniques in Therapeutic Decision-Making. Computer Aided Surgery 4, 231-247 (1999)

5. LaDisa, J.F., Guler, I., Olson, L.E., Hettrick, D.A., Kersten, J.R., Warltier, D.C., Pagel, P.S.: Three-Dimensional Computational Fluid Dynamics Modeling of Alterations in Coronary Wall Shear Stress Produced by Stent Implantation. Annals of Biomedical Engineering 31, 972-980 (2003)

6. Stuhne, G.R., Steinman, D.A.: Finite-Element Modeling of the Hemodynamics of Stented Aneurysms. Journal of Biomechanical Engineering-Transactions of the Asme 126, 382387 (2004)

7. Li, Z., Kleinstreuer, C., Farber, M.: Computational Analysis of Biomechanical Contributors to Possible Endovascular Graft Failure. Biomechanics and Modeling in Mechanobiology 4, 221-234 (2005)

8. Fung, G.S., Lam, S.K., Cheng, S.W., Chow, K.W.: On Stent-Graft Models in Thoracic Aortic Endovascular Repair: A Computational Investigation of the Hemodynamic Factors. Computers in Biology and Medicine 38, 484-489 (2008)

9. Figueroa, C.A., Taylor, C.A., Chiou, A.J., Yeh, V., Zarins, C.K.: Magnitude and Direction of Pulsatile Displacement Forces Acting on Thoracic Aortic Endografts. Journal of Endovascular Therapy 16, 350-358 (2009)

10. Wang, K.C., Dutton, R.W., Taylor, C.A.: Improving Geometric Model Construction for Blood Flow Modeling. IEEE Engineering in Medicine and Biology Magazine 18, 33-39 (1999)

11. Antiga, L., Ene-Iordache, B., Remuzzi, A.: Computational Geometry for Patient-Specific Reconstruction and Meshing of Blood Vessels from MR and CT Angiography. IEEE Transactions on Medical Imaging 22, 674-684 (2003)

12. Wilson, N.M., Arko, F.R., Taylor, C.A.: Predicting Changes in Blood Flow in PatientSpecific Operative Plans for Treating Aortoiliac Occlusive Disease. Computer Aided Surgery 10, 257-277 (2005)

13. Caselles, V., Kimmel, R., Sapiro, G.: Geodesic Active Contours. International Journal of Computer Vision 22, 61-79 (1997)

14. Boissonnat, J.D., Oudot, S.: Provably Good Sampling and Meshing of Surfaces. Graphical Models 67, 405-451 (2005)

15. Sethian, J.A.: Level Set Methods and Fast Marching Methods. Cambridge University Press, Cambridge (1999)

16. Xiong, G., Figueroa, C.A., Xiao, N., Taylor, C.A.: Simulation of Blood Flow in Deformable Arteries Using Subject-Specific Geometry and Variable Vessel Wall Properties. In: Summer Bioengineering Conference, Lake Tahoe, CA (2009)

17. Kobbelt, L.: Sqrt 3-Subdivision. In: Proceedings of the 27th Annual Conference on Computer Graphics and Interactive Techniques, pp. 103-112 (2000)

18. Desbrun, M., Meyer, M., Schröder, P., Barr, A.H.: Implicit Fairing of Irregular Meshes Using Diffusion and Curvature Flow. In: Proceedings of the 26th Annual Conference on Computer Graphics and Interactive Techniques, pp. 317-324 (1999)

19. Stanford Cardiovascular Biomechanics Lab, http://taylorlab. stanford. edu 